American Journal of Applied Sciences 6 (8): 1467-1472, 2009

ISSN 1546-9239

(C) 2009 Science Publications

\title{
Malignant Mucosal Melanoma of the Head and Neck Diagnosed in an Iranian Population over an 11-Year Period
}

\author{
Maryam Assadat Hashemi Pour, M. Rad, M.R. Zarei and G. Chamani \\ Department of Oral Medicine, Faculty of Dentistry, \\ Kerman University of Medical Sciences, Kerman, Iran \\ Member of Kerman Dental and Oral Medicine Research Center
}

\begin{abstract}
Problem statement: Mucosal Melanoma of the Head and Neck (MMHN) is a rare lethal disease. This malignancy accounts for half of all mucosal melanomas, occurring mainly in the nasal cavity, oral cavity and pharynx. They appear with equal gender distribution and with a peak incidence in the age range 60-80 years. The aim of this study was to investigate incidence, sex, age and site distribution of MMHN in the Kerman province during the time period from March 1991-2002. Approach: Documents and records of 52 patients with MMHN diagnosed from March 1991-2002 were reviewed. The patients were analyzed according to gender, age and location of the tumor. Data included in the present retrospective study were analyzed by SPSS13.5 statistical software, t-test and chi-square. Results: During this time period, 52 cases ( 25 men and 27 women) of MMHN were diagnosed. The age range was 7-84 years. The nasal cavity $(55.8 \%)$ was the most affected site. The palate and upper gingiva was the most common site in the oral cavity (8 patients, $88.8 \%$ ). Survivals 5 years in MMHN patients were 27\% and patients with mucosal melanoma of oral cavity have an $11 \%, 5$ year survival rate. Conclusion: Comparison between the findings of this study with the results obtained by other investigators showed a relative consistency.
\end{abstract}

Key words: Melanoma, malignant, head, neck

\section{INTRODUCTION}

Malignant melanoma incidence has risen markedly over the past 30-40 years and continues to increase in the United States, Canada, Australia, Asia and Europe ${ }^{[1-4]}$.

Malignant Melanoma (MM) that does not originate in the skin is a very rare tumor and is considered as one of the most deadly of all human neoplasms ${ }^{[5-8]}$. Primary mucosal melanomas of the upper aero-digestive tract are uncommon and represent only $1.7-3 \%$ of all primary melanomas ${ }^{[1-4,9,10]}$. The first case in the English literature were reported in 1885 by Lincoln: Since that time, over 1000 patients have been reviewed ${ }^{[11]}$. The most frequent localizations are the nasal fossae, the paranasal sinuses and the oral cavity where melanomas most often arise from the palate and alveolar ridge, buccal mucosa, lips, tongue and floor of mouth. MMHN comprise about $1-8 \%$ of all malignant melanomas $^{[1-4,9,10]}$ and comprise $20 \%$ of all melanomas of the head and neck melanomas ${ }^{[4,10,12]}$.

In searching the English literatures of the last 15 years for head and neck melanomas, we found very few articles that describe case series limited to mucosa of the head and neck region. Hormia and Vuori reported that mucosal melanomas represent only $0.009 \%$ of all malignant tumors is Finland ${ }^{[13]}$. Others reported that only $6.3 \%$ of all melanomas in the head and neck region are of mucosal original. According to Moore and Martin mucosal melanomas in the nasal and oral cavity account for only about $1.7 \%$ of all melanomas ${ }^{[14]}$.

Another study demonstrated that oral melanomas are very rare, accounting for only $1.6 \%$ of 7500 melanomas reported $^{[15]}$. Racial, cultural or geographic factors may predispose subjects to the this malignancy ${ }^{[16,17]}$. The mean age at diagnosis of patients with oral melanoma is 55 years (range, $40-70$ years ${ }^{[18-21]}$, although in some researches this malignancy appeared with a peak incidence in the age range $60-80$ years $^{[4,19-21]}$. A recent literature review showed the description of 14 cases of mucosal melanoma of the head and neck in a total of approximately 1000 cases of head and neck melanomas ${ }^{[22]}$. Of 14 new cases reported, only one case occurred in the oral mucosa. A high predilection was found for the palate and the maxillary gingiva with $77 \%$ of the cases reported in those sites ${ }^{[15,19-22]}$. Interestingly,

Corresponding Author: Maryam Assadat Hashemi Pour, Department of Oral Medicine, Faculty of Dentistry, Shafa St. Kerman, Iran. Member of Kerman Dental and Oral Medicine Research Center. Tel: 0983412231196/09132996183 
mucosal melanoma is much more common in Japan where it represents $22-32 \%$ of all melanomas, arising predominantly in the oral cavity ${ }^{[23]}$, although it is very rare in the white population ${ }^{[24]}$.

At a workshop recently held in Canada ${ }^{[25]}$, only 50 primary oral melanomas were retrieved from a biopsy service pool of a total of 712,000 oral biopsies from 14 institutes. The gender distribution was 74 male and $26 \%$ female, with a mean age of 56 years at diagnosis. Equal to $68 \%$ of the oral lesions were located in the palate and upper gingiva. A similar predilection for melanomas occurrence in the palate and upper gingiva has been reported in some literatures ${ }^{[10,22]}$.

Generally, survival from head and neck melanoma is reported as $17 \%$ at 5 years ${ }^{[13,21]}$ and the 10 year survival rate is $5 \%{ }^{[22]}$.

There isn't an epidemiological research about mucosal melanoma of head and neck in Iran. The aim of this article was to evaluate the prevalence of malignant melanoma of the head and neck in Kerman province, with an emphasis on the clinical manifestations of melanomas involving the oral cavity.

\section{MATERIALS AND METHODS}

Kerman is the biggest province of Iran. This study is a retrospective analysis that represents the first population-based study about the status of malignant melanoma of head and neck in Kerman province from March 1991-2002. The cancer registry in Kerman province was established in 2000 year and in the time period the present research, this center was not active. Comparison between the cancer registries with many pathological centers in the Kerman province showed that the document in cancer registry was less than that we found in pathological centers. Consequently in the data collection, the cancer center was not benefit and all data were collected from pathology records registered in 11 pathology laboratories. These laboratories receive almost all biopsy materials from Kerman province. In order to increase the accuracy of getting data in each center, the data which were obtained by two researches were checked by another two researchers with repeating the same procedure once more.

In the present research, the patients were analyzed according to sex, age and site of the tumor. Clinical, histopathologic and epidemiological data of the patients were recorded. The anatomic sites were separated into: Oral cavity, salivary glands, pharynx and nasal cavity. Cases with grossly incomplete information and repeatedly biopsies or doubly registered cases were carefully excluded from the study. This data were analyzed by SPSS13.5 statistical software, t-test and chi-square.

\section{RESULTS}

During this eleven years period, 10571 new cases of malignancy were diagnosed in Kerman province. We assessed 52 mucosal melanoma of the head and neck during 1991-2002.

This malignancy accounted for 0.49 of all malignant tumors and $6.6 \%$ of melanoma of total body (25/792) and equal to $1.9 \%$ of all cancers in the head and neck due to MMHN (52/2765). Also 35.9\% of all melanomas of the head and neck were mucosal melanoma (52/145). Age-adjusted incidence rates standardized to the world population were calculated. 52 patients were identified, yielding an age-adjusted incidence rate of 0.22 per 100000 year $^{-1}$.

The profile of the 52 patients $(25$ men and 27 women) with mucosal head and neck melanomas are shown in Table1. The relationship between sex and the appearance of malignant melanoma of head and neck wasn't statistically significant ( $p>0.05$ ).

A total of 52 cases of mucosal melanomas that involved head and neck sites were studied. It is found that, in most of the patients $(17,68 \%)$ the palatal and suprapalatal (nasal) regions were involved. The leading sites for head and neck mucosal melanoma were the nasal cavity (29 patients, 55/8\%) and the pharynx (10 patients: $19.2 \%$ ) and this was followed by oral mucosa (9 patients, 17.3\%). The gender-site relation shows that mucosal malignant melanoma of the palatalsuprapalatal regions had the same rate in men and women.

Table 2 shows the profile of the patients whose sites of involvement were those under the direct responsibility of the dental professional. The palate and upper gingiva were involved in 8 patients (88.8), 6 $(75 \%)$ of whom were men. The mean age at diagnosis was 63.5 years and the most prevalent clinical presentation in melanoma of the oral cavity was a mass. The existence of any pigmentation at the time of diagnosis was reported in $67 \%$ of the intra oral sites (Fig. 1).

All of the oral melanoma samples which were studied in the present investigation were invasive and nodular histologically. Although in non-oral melanoma samples, 67.4 were invasive and $32.6 \%$ were in situ (Table 3).

Table 1: Gender and age distribution 52 patients with mucosal melanoma of the head and neck

\begin{tabular}{lrlllll}
\hline Site & Male & Female & Mean age & Age range & $*$ M/F ratio & Total $(\%)$ \\
\hline Non-oral & 19 & 24 & 51.94 & $7-84$ & 0.79 & $43(82.7)$ \\
Oral & 6 & 3 & 64.22 & $56-72$ & 2.00 & $9(17.3)$ \\
Totals & 25 & 27 & 56.36 & $7-84$ & 0.92 & $25(100.0)$ \\
\hline
\end{tabular}

*: Male/female ratio 
Am. J. Applied Sci., 6 (8): 1467-1472, 2009

Table 2: Patients with oral mucosal melanoma

\begin{tabular}{llllll}
\hline Patient No. & Age & Gender & Site & Signs & Symptoms \\
\hline 1 & 72 & F & Hard palate & Swelling, black lump & Discomfort \\
2 & 70 & M & Hard palate & Swelling & Bleeding \\
3 & 70 & M & Soft palate & Black lump & No pain \\
4 & 60 & F & Hard palate & Swelling, 2-3 mm dark pigmented spots & Pain \\
5 & 60 & M & Maxillary ridge & Swelling & No pain \\
6 & 60 & M & Hard palate & Swelling, black lump, not fit denture & bleeding \\
7 & 56 & M & Hard palate & Swelling, black lump & Pain \\
8 & 60 & M & Hard palate & Black lump & Sore \\
9 & 70 & F & Buccal mucosa & 3 cm black and brown plaque & No pain \\
\hline
\end{tabular}

Table 3: Histological types of non-oral melanomas according to sex and location

\begin{tabular}{|c|c|c|c|c|c|c|}
\hline \multirow[b]{2}{*}{ Location } & \multicolumn{2}{|l|}{ Male } & \multicolumn{2}{|l|}{ Female } & \multicolumn{2}{|l|}{ Total } \\
\hline & In vasive & in situ & In vasive & in situ & In vasive & in situ \\
\hline Nasal cavity & 5 & 3 & 15 & 6 & 20 & 9 \\
\hline Pharynx & 2 & 3 & 3 & 2 & 5 & 5 \\
\hline $\begin{array}{l}\text { Salivary } \\
\text { glands }\end{array}$ & - & - & 4 & - & 4 & - \\
\hline Total & 7 & 6 & 22 & 8 & 29 & 14 \\
\hline
\end{tabular}

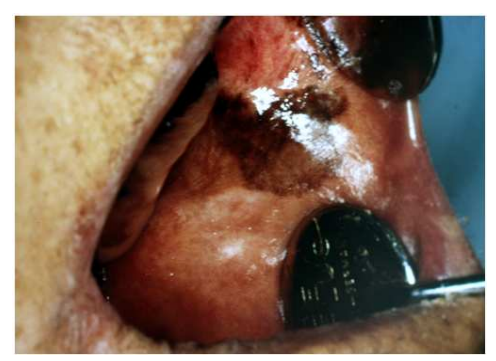

(a)

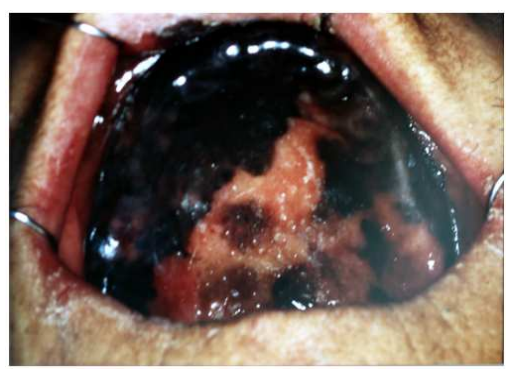

(b)

Fig. 1: ( $a$ and b): Mucosal melanoma: Pigment lesions and tumor like of the palate, gingival and the cheek

Primary treatment was surgical resection in 34 cases, in 5 cases with radiation therapy and in 12 cases chemotherapy. One patient received no treatment. 17 patients received radical neck dissection more. Four patients were treated radiation therapy and 7 patients were treated with chemotherapy after surgery.

Survivals 5 years in MMHN patients were $27 \%$. Approximately $27 \%$ of patients present with lymph node metastasis and another 24 develop lymph node metastases after treatment, whereas $12 \%$ present with distant metastasis. Over 60 of patients experience local treatment failure. Patients with mucosal melanoma of oral cavity have an $11 \%, 5$ year survival rate.

\section{DISCUSSION}

During this eleven years period, 2765 cases of head and neck cancer are diagnosed in Kerman province (approximately 251 cases of head and neck cancer each year). MMHN accounts for 1.9 of total head and neck cancers in Kerman province and intraoral melanoma accounts for only $8.8 \%$ of all oral cancers.

Unfortunately, research related to mucosal melanoma of the head and neck is limited, in as much as this disease is rare and there is a lack of large series of cases with long follow-up ${ }^{[26,27]}$. Mucosal melanoma occurs mainly between the fourth and seventh decades and most frequently in persons with 50-60 years $\operatorname{old}^{[7,8,22,28]}$. It is not clear whether there is a higher prevalence in males ${ }^{[8,22,28,29]}$. Conley reported more men with mucosal melanoma (57\%) than women with the condition $(43 \%)^{[29]}$. Although Mochimatsu et al. ${ }^{[30]}$ and Gaze et $a l^{[31]}$ reported this malignancy more in female than male ${ }^{[30,31]}$. The present study shows that $51.9 \%$ of all patients with MMHN in Kerman province were female. Also the peak of incidence in all patients was in the sixth decade. As it was seen before in many studies, it was reported that most of the patients with MMHN are female, although in many other researches, the number of males reported greater than females. About this discrepancy, several different factors can be mentioned such as: Hereditary, hormonal different.

The nasal cavity, paranasal sinuses and oral cavity are the major sites for mucosal melanoma ${ }^{[7,8,22,28,30-32]}$. In one study $49 \%$ of melanomas of the mucosal membranes of the head and neck were detected in the oral cavity ${ }^{[29]}$. In another study ${ }^{[33]}$, oral involvement was described in $37 \%$ of 38 mucosal melanomas. In our study, the most common site within head and neck region was the nasal cavity. Some studies have shown similar site distribution in the head and neck ${ }^{[7,8,30,31,33]}$. Although, it is seen in some researches that nasal cavity and oral cavity are reported as the most common site $^{[4,22,28]}$. 
Gorsky et al. ${ }^{[8]}$ investigated the six oral melanomas samples and it was found that three patients (50) had asymptomatic swelling and one of the patients had bleeding(16.6\%). Only one patient (16.6) had pain and pigmentation was observed in 2 patients (33.3\%). In three cases $(50 \%)$ of the oral melanomas, tumor was located in the palate ${ }^{[8]}$.

Because most of the mucosal melanotic lesions are painless in their early stages, the diagnosis is unfortunately often delayed until symptoms resulting from ulceration, growth and/or bleeding are noted ${ }^{[22]}$.

Because of the possibility of the development of oral melanoma within pigmented areas ${ }^{[22]}$, such areas should be biopsied. The oral lesions in our group were most frequently diagnosed after identification of a mass: only $33 \%$ of the patients complained of pain or discomfort. In clinical appearance, $67 \%$ of these lesions were described as pigmented, although others have reported that pigmentation is present only in approximately one third of patients ${ }^{[18,33]}$. Therefore biopsies should be performed in all pigmented lesions that are recently occurred or on the lesions those are suspected about their clinical appearance. Also, all pigmented lesions which are not consistent with amalgam tattoo or physiologic pigmentation must be biopsies. Certainly, biopsy is essential in suspected lesions and should not be delayed ${ }^{[8]}$.

It is noteworthy that nearly one-third of mucosal melanomas in the head and neck region are amelanotic $^{[34]}$. Also in our study, 23\% of MMHN were not pigmented. Early detection of mucosal melanoma is further complicated when the lesion is not pigmented (amelanotic), which in some series is true of most of the lesions subsequently diagnosed as melanoma. Therefore, any growing lesion, pigmented or nonpigmented, requires that a diagnosis be made and it should be biopsied without delay. It may be appropriate to excise small masses, but large lesions should be sampled with incisional techniques ${ }^{[8]}$.

The treatment of choice for mucosal melanoma, like cutaneous melanoma, is complete excision with adequate negative margins ${ }^{[26,35]}$, which entails complete resection of the primary tumor and any positive cervical lymph nodes. The likelihood of local recurrence after resection is approximately $50 \%{ }^{[26]}$. The role of radiotherapy as primary management of mucosal malignant melanoma remains controversial, but its use following surgery does seem to achieve local tumor control and patient survival ${ }^{[36]}$. The efficacy of adjuvant systemic therapy, such as interferon and vaccine therapy, remains investigational for patients with unfavorable cutaneous melanomas ${ }^{[26]}$. In present study 34 cases were treatment by surgery.
MMHN carry a poor outcome and the impact of different treatments on survival is not yet clearly ${ }^{[7,37]}$. The 5 year survival rates vary from approximately 20$50 \%$. Although the median time to relapse is roughly 1 year or less, late failures are common and causespecific survival continues to decline after 5 years ${ }^{[27]}$. Bartell et al. ${ }^{[38]}$ show that the median time to disease progression for all 15 patients was 10 months. The median overall survival duration for all patients was 22 months $^{[38]}$. Also Yanagi et al. ${ }^{[39]}$ show that the 5 year overall and cause-specific survival rates were 27.0 and $39.6 \%$ respectively ${ }^{[39]}$. In our study survivals 5 years in MMHN patients were $27 \%$.

The suspected signs of oral melanoma include swelling, ulceration, pigmentation, discomfort or painful sensation, bleeding, ill-fitting dentures and any combination of the above $\mathrm{e}^{[4,19-22]}$. In this present study signs of MMHN were swelling, pigmentation, discomfort and painful sensation.

\section{CONCLUSION}

Despite a considerable volume of literature written about MMHN in many countries, the incidence of this cancer hasn't as yet been thoroughly documented or analyzed in Iran. Therefore it is important for our oral medicine and otolaryngology specialists to be aware of the incidence rate and clinical presentation of mucosal melanoma of the head and neck. Also they should be aware that mucosal melanoma has different behavior, treatment and prognosis with respect to its cutaneous counterpart.

\section{REFERENCES}

1. Greenberg, M.S. and KM. Glic, 2008. Burket's Oral Medicine. 11th Edn., BC Decker, Hamilton, ISBN: 1550093452, pp: 586.

2. Prabhu, S.R., D.F. Wilson and D.K. Daftary, 1992. Oral Diseases in the Tropics. Oxford University Press, New York, ISBN: 10: 0192620088, pp: 824.

3. Neville, B.W., D. Damm, R. Allenc and J.E. Bouquot, 2008. Oral and Maxillofacial Pathology. 3rd Edn., W.B. Saunders, Philadelphia, pp: 376-380.

4. Lengyel, E., K. Gilde, E. Remenar and O. Esik, 2003. Malignant mucosal melanoma of the head and neck-a review. Pathol. Oncol. Res., 9: 7-12. http://direct.bl.uk/research/32/03/RN131499097.ht $\mathrm{ml}$ ? source $=$ googlescholar

5. Loree, T.R., A.P. Mullins, J. Spellman, J.H. North and W.L. Hicks, 1999. Head and neck mucosal melanoma: A 32-year review. Ear Nose Throat J., 78: 372-375.

http://www.ncbi.nlm.nih.gov/pubmed/10355199 
6. Medina, J.E., A. Ferlito, P.K. Pellitteri, A.R. Shaha, A. Khafif and K.O. Devaney et al., 2003. Current management of mucosal melanoma of the head and neck. J. Surg. Oncol., 83: 116-122. http://www.ncbi.nlm.nih.gov/pubmed/12772206

7. Penel, N., Y. Mallet, X. Mirabel, J.T. Van and J.L. Lefebvre, 2006. Primary mucosal melanoma of head and neck: Prognostic value of clear margins. Laryngo, 116: 993-995. http://www.ncbi.nlm.nih.gov/pubmed/16735914

8. Gorsky, M. and J.B. Epstein, 1998. Melanoma arising from the mucosal surfaces of the head and neck. Oral Surg. Oral. Med. Oral. Pathol. Oral. Radiol. $\quad$ Endod., $\quad 86: \quad 715-719$. http://www.ncbi.nlm.nih.gov/pubmed/9868730

9. Calabrese, V., M. Cifola, R. Pareschi, A. Parma and A. Sonzogni, 1989. Primary malignant melanoma of the oral cavity. J. Laryngol. Otol., 103: 887-889. DOI: 10.1017/S0022215100110412

10. Disky, A., D. Campos and H. Benchikhi, 2008. Case report: Mucosal melanoma of the lip and the cheek. Dermatol. J., 14: 20-20. http://www.ncbi.nlm.nih.gov/pubmed/19061578

11. Batsakis, J.D., J.A. Regezi and A.R. Solomon, 1982. The pathology of head and neck tumors-part 13: Mucosal melanomas. Head Neck Surg., 4: 404-412. http://www.ncbi.nlm.nih.gov/pubmed/7096100

12. Matthew, A., M.D. Kienstra, A.L. Tapan and M.D. Padhya, 2005. Head and neck melanoma. Cancer Control, 12: 242-247. https://www.moffitt.org/moffittapps/ccj/v12n4/pdf/ 242.pdf

13. Homia, M. and E.J. Vuori, 1969. Mucosal melanomas of the head and neck. J. Laryngol. Otol., 83: 349-59.

14. Moore, E.S. and H. Marton, 1955. Melanoma of the upper respiratory tract and oral cavity. Cancer, 8: $1167-1176$. http://www.ncbi.nlm.nih.gov/pubmed/13270234

15. Pliskin, M.E., 1979. Malignant Melanoma of the Oral Cavity. In: Human Malignant Melanoma, Clarck, W.H. Jr, L.I. Goldman and M.J. Mastranhelo (Eds.). Grune and Stallon, New York. pp: 34-45, 78,125

16. Takagi, M., G. Ishikawa and W. Mori, 1974. Primary malignant melanoma of the oral cavity in Japan: With special reference to mucosal melanomas. Cancer, 34: 358-370.

17. Umeda, M., Y. Mishima, O. Teranobu, K. Nakanishi and K. Shimada, 1988. Heterogeneity of primary malignant melanomas in oral mucosa an analysis of 43 cases in Japan. Pathology, 20: 234-241. http://www.ncbi.nlm.nih.gov/pubmed/3060820
18. Macintyre, D.R. and J.C. Briggs, 1984. Primary malignant melanoma. Int. J. Oral. Surg., 13: 160-165. http://cat.inist.fr/?aModele=afficheN\&cpsidt $=8924989$

19. Hashemi, P.M.S., 2008. Malignant melanoma of the oral cavity: A review of literature. Ind. J. Dent. Res., 19: 47-51. http://www.ijdr.in/article.asp?issn=09709290; year $=2008$; volume $=19 ;$ issue $=1 ;$ spage $=47$; epa ge $=51$; aulast $=$ Hashemi

20. Zarei, M.R., G. Chamani and M.S. Hashemipour, 2005. Epidemiological aspects of non-squamous cell malignant tumors of the oral cavity in Iranians. Oral Oncol., 41: 141. DOI: 10.1016/j.oraloncology.2005.04.019

21. Zarei, M.R., G. Chamani and M.S. Hashemipour, 2007. Epidemiological aspects of non-squamous cell malignant tumors of the oral cavity and pharynx in Iranians. J. Dent. TUMS., 4: 34-45. http://journals.tums.ac.ir/upload_files/pdf/3963.pdf

22. Anneroth, G., G.O. Carlson, C.M. Eneroth and G. Moberger, 1973. Primary melanoma in the oral mucous membrane. Swed. Dent. J., 66: 27-37. http://www.ncbi.nlm.nih.gov/pubmed/4569627

23. Mody, R.N. and S.V. Puranik, 1992. Oral malignant melanoma. A case report. Ind. J. Dent. Res., 3: 121-122. http://www.ncbi.nlm.nih.gov/pubmed/1344982

24. Manolidis, S. and P.J. Donald, 1997. Malignant mucosal melanoma of the head and neck: Review of the literature and report of the 14 patients. Cancer, $\quad 80: \quad 1373-1386$. http://www.ncbi.nlm.nih.gov/pubmed/9338460

25. Barker, B.F., W.M. Carpenter, T.E. Daniels, M.A. Kahn, A.S. Leider and F. Lozada-Nur et al., 1997. Oral mucasal melanomas: The WESTOP Banff workshop proceedings. Oral Surg. Oral Med. Oral Pathol., 83: 672-679. http://www.ncbi.nlm.nih.gov/pubmed/9195622

26. Meleti, M., C.R. Leemans, R. De Bree, P. Vescovi, E. Sesenna and I. Vander Waal, 2008. Head and neck mucosal melanoma: Experience with 42 patients, with emphasis on the role of postoperative radiotherapy. Head Neck, 30: 1543-1551. http://www.ncbi.nlm.nih.gov/pubmed/18704960

27. Mendenhall, W.M., R.J. Amdur, R.W. Hinerman, J.W. Werning, D.B. Villaret and N.P. Mendenhall, 2005. Head and neck mucosal melanoma. Am. J. Clin. Oncol., 28: 626-30. http://www.oralcancerfoundation.org/facts/pdf/mel anoma.pdf

28. Chiu N.T., M.A. Weinstock, 1996. Melanoma of oronasal mucosa. Population-based analysis of occurrence and mortality. Arch Otolaryngol Head Neck Surg., $\quad$ 122: 985-988. http://www.ncbi.nlm.nih.gov/pubmed/ 8797564 
29. Conley, J., 1989. Melanoma of the mucous membrane of the head and neck. Laryngoscope, 99: $1248-1254$. http://www.ncbi.nlm.nih.gov/pubmed/2601538

30. Mochimatsu, I., M. Tsukuda, M. Kurihara, H. Enomoto, Y. Kagesato, S. Kawai and Z.L. Xin, 1996. Case reports of mucosal melanoma of the head and neck. Nippon Jibiinkoka Gakkai Kaiho, 99: 552-557. http://www.ncbi.nlm.nih.gov/pubmed/8683365

31. Gaze, M.N., G.R. Kerr and J.F. Smyth, 1990. Mucosal melanomas of the head and neck: The scottish experience. The Scottish melanoma group. Clin. Oncol., 2: 277-83. http://www.ncbi.nlm.nih.gov/pubmed/2261428

32. McLean, N., M. Tighiouart and S. Muller, 2008. Primary mucosal melanoma of the head and neck. Comparison of clinical presentation and histopathologic features of oral and sinonasal melanoma. Oral Oncol., 44: 1039-1046. DOI: 10.1016/j.oraloncology.2008.01.014

33. Berthelsen, A.A.P. Andersen, T.S. Jensen and H.S. Hansen, 1984. Melanomas of the mucosa in the oral cavity and upper respiratory passages. Cancer, 54: 907-912. http://www.ncbi.nlm.nih.gov/pubmed/6744219

34. Gilligan, D. and N.J. Slevin, 1991. Radical radiotherapy for 28 cases of mucosal melanoma in the nasal cavity and sinuses. Br. J. Radiol., 64: 1147-1150.

http://www.ncbi.nlm.nih.gov/pubmed/1773274
35. Krengli, M., B.A.J. Fossa, J.H. Kaanders, L. Masini, D. Beldì and R. Orecchia, 2008. What is the role of radiotherapy in the treatment of mucosal melanoma of the head and neck? Crit. Rev. Oncol. Hematol., 65:

121-128. http://www.ncbi.nlm.nih.gov/pubmed/17822915

36. Bradley, P.J., 2006. Primary malignant mucosal melanoma of the head and neck. Curr. Opin. Otolaryngol. Head Neck Surg., 14: 100-104. http://www.ncbi.nlm.nih.gov/pubmed/16552267

37. Teppo, H., J. Kervinen, P. Koivunen and O.P. Alho, 2006. Incidence and outcome of head and neck mucosal melanoma-a population-based survey from Northern Finland. Int. J. Circumpolar. Health, 65: 443-447. http://www.ncbi.nlm.nih.gov/pubmed/17319088

38. Bartell, H.L., A.Y. Bedikian, N.E. Papadopoulos, T.K. Dett, M.T. Ballo and J.N. Myers et al., 2008. Biochemotherapy in patients with advanced head and neck mucosal melanoma. Head Neck, 30: 1592-1598. http://www.ncbi.nlm.nih.gov/pubmed/18798304

39. Yanagi, T., J.E. Mizoe, A. Hasegawa, R. Takagi, H. Bessho and T. Onda et al., 2008. Mucosal malignant melanoma of the head and neck treated by carbon ion radiotherapy. Int. J. Radiat. Oncol. Biol. Phys., 74: 15-20. DOI: 10.1016/j.ijrobp.2008.07.056 